UT-Komaba/11-10

\title{
Signatures of low-scale string models at the LHC
}

\author{
Manami Hashi ${ }^{a}$ and Noriaki Kitazawa ${ }^{b}$ \\ ${ }^{a}$ Institute of Physics, University of Tokyo, \\ Komaba 3-8-1, Meguro-ku, Tokyo 153-8902, Japan \\ e-mail: hashi@hep1.c.u-tokyo.ac.jp \\ ${ }^{b}$ Department of Physics, Tokyo Metropolitan University, \\ Hachioji, Tokyo 192-0397, Japan \\ e-mail: kitazawa@phys.se.tmu.ac.jp
}

\begin{abstract}
Low-scale string models, in which the string scale $M_{\mathrm{s}}$ is of the order of $\mathrm{TeV}$ with large extra dimensions, can solve the problems of scale hierarchy and non-renormalizable quantum gravity in the standard model. String excited states of the standard model particles are possibly observed as resonances in the dijet invariant mass distribution at the LHC. There are two properties to distinguish whether the resonances are due to low-scale string or some other "new physics". One is a characteristic angular distribution in dijet events at the resonance due to spin degeneracy of string excited states, and the other is an appearance of the second resonance at a characteristic mass of second string excited states. We investigate a possibility to observe these evidences of low-scale string models by Monte Carlo simulations with a reference value of $M_{\mathrm{s}}=4 \mathrm{TeV}$ at $\sqrt{s}=14 \mathrm{TeV}$. It is shown that spin degeneracy at the dijet resonance can be observed by looking the $\chi$-distribution with integrated luminosity of $20 \mathrm{fb}^{-1}$. It is shown that the second resonance can be observed at rather close to the first resonance in the dijet invariant mass distribution with integrated luminosity of $50 \mathrm{fb}^{-1}$. These are inevitable signatures of low-scale string models.
\end{abstract}




\section{Contents}

1 Introduction $\quad 1$

2 The first string resonances

3 Angular analysis

4 The second string resonances $\quad 10$

5 Conclusions

A Amplitudes and widths with string excited states

\section{Introduction}

One of theoretical problems of the Standard Model (SM) is that it does not describe gravitational interaction in a renormalizable form. String Theory is a strong candidate for a theory of quantum gravity. There is another theoretical problem so-called hierarchy problem. If the fundamental energy scale of gravitational interaction, i.e., the Planck scale, is considered as a fundamental scale of the SM, a large hierarchy between the Planck scale $M_{\mathrm{Pl}} \sim 10^{19} \mathrm{GeV}$ and the weak scale $M_{\mathrm{W}} \sim 10^{2} \mathrm{GeV}$ can not be understood. It is proposed that the string scale $M_{\mathrm{s}}=\sqrt{1 / \alpha^{\prime}}$ which is the fundamental scale in String Theory could be much lower than the Planck scale due to the existence of large extra dimensions [1, 2]. This is a solution to the hierarchy problem in the framework of String Theory with the string scale $M_{\mathrm{s}} \sim \mathcal{O}(1) \mathrm{TeV}$. Such string models with the low string scale are called low-scale string models (for review, see Ref.[3]).

In low-scale string models, the Planck scale is described as

$$
M_{\mathrm{Pl}}^{2}=\frac{8}{g_{\mathrm{s}}^{2}} M_{\mathrm{s}}^{8} \frac{V_{6}}{(2 \pi)^{6}},
$$

where $g_{\mathrm{s}}$ is string coupling and $V_{6}$ is the volume of six-dimensional compactified space. Note that closed strings which mediate gravitational interaction propagate in whole ten-dimensional space-time. If it is assumed that $g_{\mathrm{s}}$ is small for perturbative theory, $V_{6} M_{\mathrm{s}}^{6} \sim 10^{32}$ is required with $M_{\mathrm{s}} \sim \mathcal{O}(1) \mathrm{TeV}$. Imagine a $\mathrm{D} p$-brane whose $p$-dimensional space contains our three-dimensional space. Open strings on the $\mathrm{D} p$-brane give gauge symmetry, and the gauge coupling constant in our four-dimensional space-time is given by

$$
\left(\frac{g^{2}}{4 \pi}\right)^{-1}=\frac{2}{g_{\mathrm{s}}} M_{\mathrm{s}}^{p-3} \frac{V_{p-3}}{(2 \pi)^{p-3}}
$$

where $V_{p-3}$ is the volume of $(p-3)$-dimensional compactified space parallel to the $\mathrm{D} p$-brane. For appropriately large gauge coupling, $V_{p-3}$ should not be very large: $V_{p-3} M_{s}^{p-3} \sim 1$. With the above condition for large $M_{\mathrm{Pl}}$, the volume of $(9-p)$-dimensional compactified space, $V_{9-p}$, should be large as much as $V_{9-p} M_{s}^{9-p} \sim 10^{32}$, because $V_{6}=V_{p-3} \times V_{9-p}$. Gravity escapes to 
large transverse directions to the $\mathrm{D} p$-brane and the strength of gravity on the $\mathrm{D} p$-brane becomes weak. It has been shown in Ref. [4 that this type of anisotropic compactification is possible in String Theory.

A gauge symmetry $\mathrm{U}(N)$ is realized on a stack of $N$ D-branes. We consider models in which such stacks of D-branes relevant to the SM are localized in compactified space, i.e., "local models" in Ref.[5]. We need, for example, four stacks of D-branes to realize $\mathrm{U}(3)_{\text {color }} \times \mathrm{U}(2)_{\text {left }} \times \mathrm{U}(1) \times \mathrm{U}(1)^{\prime}$ gauge symmetry. Since $\mathrm{U}(N)=\mathrm{SU}(N) \times \mathrm{U}(1)$, there is an $\mathrm{U}(1)$ symmetry on each stack of D-branes. The massless modes of open strings on $U(3)_{\text {color }}$ branes are identified as gluons and an additional $\mathrm{U}(1)$ color gauge boson, and the massless modes of open strings on $\mathrm{U}(2)_{\text {left }}$ branes are identified as weak bosons and an additional $\mathrm{U}(1)_{\text {left }}$ gauge boson. The $\mathrm{U}(1) \mathrm{Y}$ gauge symmetry in the $\mathrm{SM}$ is an independent linear combination of four $\mathrm{U}(1)$ symmetries 1

The chiral matter for the SM is realized by open strings whose two ends attach on two different stacks of D-branes intersecting with each other [6]. Space of the intersection between the two different stacks contains our three-dimensional space and the open strings are localized on that space. For example, left-handed quark doublets $Q_{\mathrm{L}}$ are realized by the massless modes of open strings between $\mathrm{U}(3)_{\text {color }}$ and $\mathrm{U}(2)_{\text {left }}$ branes, right-handed up-type quarks $\bar{U}_{\mathrm{R}}$ are realized by the massless modes of open strings between $\mathrm{U}(3)_{\text {color }}$ and $\mathrm{U}(1)$ branes, and so on.

In addition to the SM particles, there are many massive states: "string excited states" as massive modes of open strings, Kaluza-Klein (KK) states of the SM particles and string excited states, and various closed string states.

In this paper, we explore a possibility to observe signatures of low-scale string models in dijet events at the LHC. As it is reviewed in the rest of this section, there are some modelindependent features in parton two-body scattering amplitudes. We investigate dijet events including contributions of string models by Monte Carlo simulations. We concentrate on the following two distinct properties.

- A resonance in the dijet invariant mass distribution by "string excited states" consists many degenerated states with various spins. An analysis of the dijet angular distribution at the resonance must be very important to have a signature of low-scale string models [7, 8, 9].

- String $n$th excited states have masses of $M_{n}=\sqrt{n} M_{\mathrm{s}}$. A second resonance at a place $\sqrt{2}$ times far from the place of the first resonance in the dijet invariant mass distribution, is a signature of low-scale string models.

In the rest of this section, we discuss the model independency of predictions to parton two-body scatterings at the LHC by low-scale string models [5]. We begin with summarizing generally spectra of low-scale string models in four-dimensional space-time.

The spectra of excited states of open strings can be understood from open-string two-body scattering amplitudes which are calculated by the world-sheet superconformal field theory in flat space-time. If we consider a D3-brane where the world volume coincides with our four dimensional space-time, the open-string amplitudes depend only on the string scale $M_{\mathrm{s}}$, group

\footnotetext{
${ }^{1}$ Remaining three U(1) symmetries are usually "anomalous", and these gauge bosons are massive.
} 
theoretical factor and gauge coupling constant, and do not depend on the details of model buildings such as the way of compactification and configuration of D-branes. String effects in the open-string two-body scattering amplitudes are described by "string form factors" which are functions of the Mandelstam variables $s, t$ and $u$ (with $s+t+u=0$ ) [10]. A typical form of the form factor function is

$$
V(s, t, u)=\frac{\Gamma\left(1-s / M_{\mathrm{s}}^{2}\right) \Gamma\left(1-u / M_{\mathrm{s}}^{2}\right)}{\Gamma\left(1+t / M_{\mathrm{s}}^{2}\right)} .
$$

In a low-energy limit $M_{\mathrm{s}} \rightarrow \infty$, the string effects disappear as $V(s, t, u) \rightarrow 1$, and the string amplitudes become equal to the SM amplitudes (see Appendix A for details). In case of a finite value for $M_{\mathrm{s}}$, the form factor function is expanded by a sum over infinite $s$-channel poles,

$$
V(s, t, u) \simeq \sum_{n=1}^{\infty} \frac{1}{(n-1) !} \frac{1}{\left(M_{\mathrm{s}}^{2}\right)^{n-1}} \frac{1}{s-n M_{\mathrm{s}}^{2}} \prod_{J=0}^{n-1}\left(u+J M_{\mathrm{s}}^{2}\right)
$$

This expansion is a good approximation, near each of $n$th pole $s \simeq n M_{\mathrm{s}}^{2}$. These poles correspond to string excited states which have masses $M_{n}=\sqrt{n} M_{\mathrm{s}}$. They are degenerated with different spins from $J=j_{0}$ to $J=j_{0}+(n-1)$ for each $n$th pole, where $j_{0}$ is an original spin of initial states in the two-body scattering processes. These string excited states are exchanged as virtual states in $s$-channel and are experimentally observed as resonances in these processes of the SM particles. Signals by the string excited states at colliders were originally pointed out in Ref.[10].

If open strings have momenta and windings around in the direction of extra dimensions with general $\mathrm{D} p$-branes $(p>3)$, KK modes and winding modes of open strings appear in our four-dimensional space-time. The masses and the ways to contribute to amplitudes of KK and winding modes depend on the details of the way of compactification. Typical masses of quantized KK modes and winding modes are $M_{n}^{\mathrm{KK}}=n\left(V_{p-3}\right)^{-\frac{1}{p-3}}$ and $M_{n}^{\text {wind. }}=n M_{\mathrm{s}}^{2}\left(V_{p-3}\right)^{\frac{1}{p-3}}$, respectively. Their masses approximate $n M_{\mathrm{s}}$ and they are different from the masses of $n$th string excited states, $M_{n}=\sqrt{n} M_{\mathrm{s}}$.

Since closed string states interact only at one-loop level with the SM particles due to openclosed string duality, we do not consider these states. Black holes may also be produced because of the low string scale, namely, the low fundamental gravitational scale. However, since these produced black holes are expected to be evaporated by Hawking radiation, they do not give contributions to dijet events. We do not consider black holes also.

We concentrate on dijet events which are caused by parton two-body scattering processes at the LHC. Since a target is the physics on resonances in the dijet invariant mass distribution, we may only discuss model independency of states which give $s$-channel poles in the scattering amplitudes. If momenta in the direction of extra dimensions are conserved at interaction vertices, states of KK modes (winding modes) can appear only in pair and do not contribute to $s$-channel poles. Momenta in the direction transverse to D-branes are non-conserved and momenta in the direction parallel to D-branes are conserved. Therefore, even if D-branes contain extra dimensions, there are no $s$-channel exchanges of single KK modes (winding modes) in scattering processes of open strings on the D-branes. In scattering processes of open strings connecting two different stacks of D-branes, however, there are $s$-channel exchanges of single KK modes (winding modes) due to non-conservation of momenta. 

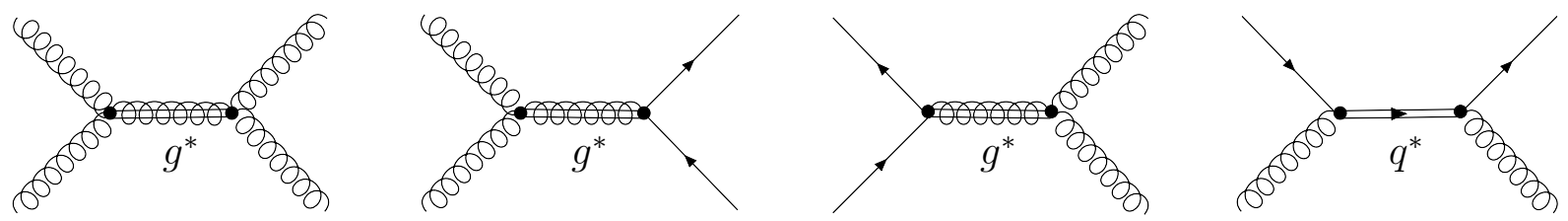

Figure 1.1: The model independent two-parton scattering processes with exchanges of string excited states of gluons and quarks, $g^{*}$ and $q^{*}$, respectively.

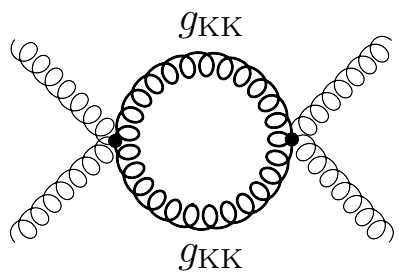

Figure 1.2: The $g g \rightarrow g g$ process with the exchange of a pair of KK gluons $g_{\mathrm{KK}}$.
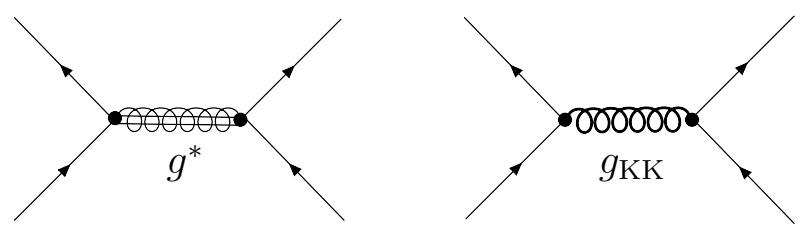

Figure 1.3: The model-dependent processes with the exchange of a KK gluon $g_{\mathrm{KK}}$.

The processes of $g g \rightarrow g g, g g \rightarrow q \bar{q}$ and $q \bar{q} \rightarrow g g$ are model independent. In these processes, only string excited states of gluons can contribute to $s$-channel poles as shown in Fig,1.1, and KK modes (winding modes) of gluons can appear only in pair as shown in Fig, 1.2. The process of $q g \rightarrow q g$ is also model independent with string excited states of quarks in $s$-channel as shown in Fig.1.1, because momenta on the intersection plane where open strings of chiral fermions are localized are conserved. On the other hand, the process of $q \bar{q} \rightarrow q \bar{q}$ is model dependent, since both of KK modes (winding modes) of gluons and gluon excitations are exchanged in $s$-channel as shown in Fig.1.3. Fortunately, cross sections of this process are suppressed by the effect of parton distribution functions (PDFs) at the LHC as a proton-proton collider. We concentrate on the above model-independent processes at the LHC, and discuss leading string signals which do not depend on the details of model buildings.

In Section 2, the appearance of the resonance in the dijet invariant mass distribution due to first string excited states is reviewed. In Section 3, it is shown that spin degeneracy at the resonance can be observed by analyzing the dijet angular distribution. In Section 4, it is shown that the second resonance can be observed at rather close to the first resonance in the dijet invariant mass distribution. In Section 5, we give a summary of our results and some discussions. Some detailed properties of amplitudes and widths of first and second string excited states are summarized in Appendix A. 


\section{The first string resonances}

Spin-averaged squared amplitudes of the model-independent parton two-body scattering processes with exchanges of the first string excited states are calculated in Ref. [7, 8, 11].

$$
\begin{aligned}
& \left|\mathcal{M}_{1 \mathrm{st}}(g g \rightarrow g g)\right|^{2}=\frac{8}{N^{2}} \frac{g^{4}}{M_{\mathrm{s}}^{4}}\left\{\frac{\left(N^{2}-4\right)^{2}}{4\left(N^{2}-1\right)}\left[\frac{M_{\mathrm{s}}^{8}}{\left(\hat{s}-M_{\mathrm{s}}^{2}\right)^{2}+\left(M_{\mathrm{s}} \Gamma_{g^{*}, 1 \mathrm{st}}^{J=0}\right)^{2}}+\frac{\hat{u}^{4}+\hat{t}^{4}}{\left(\hat{s}-M_{\mathrm{s}}^{2}\right)^{2}+\left(M_{\mathrm{s}} \Gamma_{g^{*}, 1 \mathrm{st}}^{J=2}\right)^{2}}\right]\right. \\
& \left.+\left[\frac{M_{\mathrm{s}}^{8}}{\left(\hat{s}-M_{\mathrm{s}}^{2}\right)^{2}+\left(M_{\mathrm{s}} \Gamma_{C^{*}, 1 \mathrm{st}}^{J=0}\right)^{2}}+\frac{\hat{u}^{4}+\hat{t}^{4}}{\left(\hat{s}-M_{\mathrm{s}}^{2}\right)^{2}+\left(M_{\mathrm{s}} \Gamma_{C^{*}, 1 \mathrm{st}}^{J=2}\right)^{2}}\right]\right\}, \\
& \left|\mathcal{M}_{1 \mathrm{st}}(g g \rightarrow q \bar{q})\right|^{2}=\frac{2}{N\left(N^{2}-1\right)} N_{f} \frac{g^{4}}{M_{\mathrm{s}}^{4}}\left[\frac{N^{2}-4}{2} \frac{\hat{u} \hat{t}\left(\hat{u}^{2}+\hat{t}^{2}\right)}{\left(\hat{s}-M_{\mathrm{s}}^{2}\right)^{2}+\left(M_{\mathrm{s}} \Gamma_{g^{*}, 1 \mathrm{st}}^{J=2}\right)^{2}}+\frac{\hat{u} \hat{t}\left(\hat{u}^{2}+\hat{t}^{2}\right)}{\left(\hat{s}-M_{\mathrm{s}}^{2}\right)^{2}+\left(M_{\mathrm{s}} \Gamma_{C^{*}, 1 \mathrm{st}}^{J}\right)^{2}}\right] \\
& \left|\mathcal{M}_{1 \mathrm{st}}(q \bar{q} \rightarrow g g)\right|^{2}=\frac{2\left(N^{2}-1\right)}{N^{3}} \frac{g^{4}}{M_{\mathrm{s}}^{4}}\left[\frac{N^{2}-4}{2} \frac{\hat{u} \hat{t}\left(\hat{u}^{2}+\hat{t}^{2}\right)}{\left(\hat{s}-M_{\mathrm{s}}^{2}\right)^{2}+\left(M_{\mathrm{s}} \Gamma_{g^{*}, 1 \mathrm{st}}^{J}\right)^{2}}+\frac{\hat{u} \hat{t}\left(\hat{u}^{2}+\hat{t}^{2}\right)}{\left(\hat{s}-M_{\mathrm{s}}^{2}\right)^{2}+\left(M_{\mathrm{s}} \Gamma_{C^{*}, 1 \mathrm{st}}^{J=2}\right)^{2}}\right], \\
& \left|\mathcal{M}_{1 \mathrm{st}}(q g \rightarrow q g)\right|^{2}=\left|\mathcal{M}_{1 \mathrm{st}}(\bar{q} g \rightarrow \bar{q} g)\right|^{2} \\
& =\frac{N^{2}-1}{2 N^{2}} \frac{g^{4}}{M_{\mathrm{s}}^{2}}\left[\frac{M_{\mathrm{s}}^{4}(-\hat{u})}{\left(\hat{s}-M_{\mathrm{s}}^{2}\right)^{2}+\left(M_{\mathrm{s}} \Gamma_{q^{*}, 1 \mathrm{st}}^{J=1 / 2}\right)^{2}}+\frac{(-\hat{u})^{3}}{\left(\hat{s}-M_{\mathrm{s}}^{2}\right)^{2}+\left(M_{\mathrm{s}} \Gamma_{q^{*}, 1 \mathrm{st}}^{J=3 / 2}\right)^{2}}\right] \text {, } \\
& \left|\mathcal{M}_{1 \mathrm{st}}(q g \rightarrow g q)\right|^{2}=\left|\mathcal{M}_{1 \mathrm{st}}(\bar{q} g \rightarrow g \bar{q})\right|^{2} \\
& =\frac{N^{2}-1}{2 N^{2}} \frac{g^{4}}{M_{\mathrm{s}}^{2}}\left[\frac{M_{\mathrm{s}}^{4}(-\hat{t})}{\left(\hat{s}-M_{\mathrm{s}}^{2}\right)^{2}+\left(M_{\mathrm{s}} \Gamma_{q^{*}, 1 \mathrm{st}}^{J=1 / 2}\right)^{2}}+\frac{(-\hat{t})^{3}}{\left(\hat{s}-M_{\mathrm{s}}^{2}\right)^{2}+\left(M_{\mathrm{s}} \Gamma_{q^{*}, 1 \mathrm{st}}^{J=3 / 2}\right)^{2}}\right],
\end{aligned}
$$

where $N=3, N_{f}=6$ and $\Gamma_{g^{*}, C^{*}, q^{*}}^{J}$ are total decay widths of the first excited states of gluons, the $\mathrm{U}(1)_{\text {color }}$ gauge boson and quarks with spin $J$, respectively,

$$
\begin{array}{ll}
\Gamma_{g^{*}, 1 \mathrm{st}}^{J=0}=\frac{g^{2}}{4 \pi} M_{\mathrm{s}} \frac{N}{4}, & \Gamma_{g^{*}, 1 \mathrm{st}}^{J=2}=\frac{g^{2}}{4 \pi} M_{\mathrm{s}}\left(\frac{N}{10}+\frac{N_{f}}{40}\right), \\
\Gamma_{C^{*}, 1 \mathrm{st}}^{J=0}=\frac{g^{2}}{4 \pi} M_{\mathrm{s}} \frac{N}{2}, & \Gamma_{C^{*}, 1 \mathrm{st}}^{J=2}=\frac{g^{2}}{4 \pi} M_{\mathrm{s}}\left(\frac{N}{5}+\frac{N_{f}}{40}\right), \\
\Gamma_{q^{*}, 1 \mathrm{st}}^{J=1 / 2}=\frac{g^{2}}{4 \pi} M_{\mathrm{s}} \frac{N}{8}, & \Gamma_{q^{*}, 1 \mathrm{st}}^{J=3 / 2}=\frac{g^{2}}{4 \pi} M_{\mathrm{s}} \frac{N}{16},
\end{array}
$$

where $g$ is the gauge coupling constant of strong interaction 2 . Here, $\hat{s}, \hat{t}$ and $\hat{u}$ are the Mandelstam variables of partons. The squared amplitudes of eqs.(2.1)-(2.5) are "minimal", because we do not consider contributions in $\hat{t}$ - and $\hat{u}$-channels, which should be a good approximation for any low-scale string models at least near the poles of first string excited states, $\hat{s} \simeq M_{\mathrm{s}}^{2}$.

\footnotetext{
${ }^{2}$ The value of $\Gamma_{q^{*}}^{J=3 / 2}$ is different by factor $1 / 2$ from the one in Ref.[11]. See Appendix $\mathrm{A}$ for details.
} 


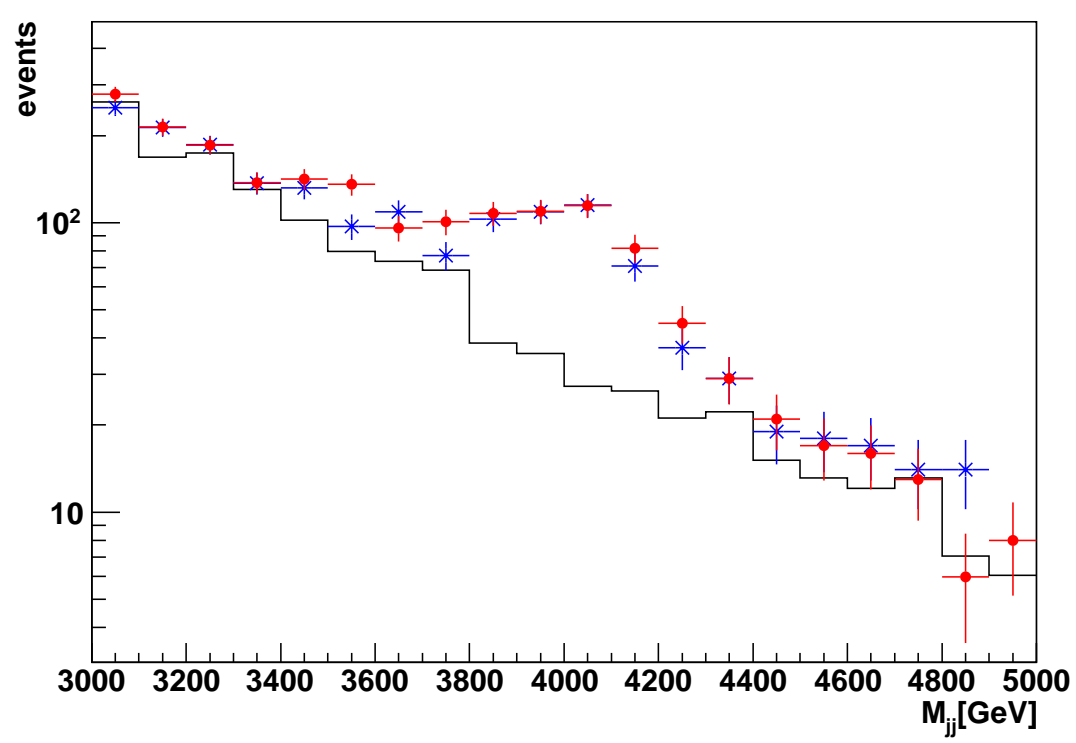

Figure 2.1: The dijet invariant mass distribution for $M_{\mathrm{s}}=4 \mathrm{TeV}$ with $1.4 \mathrm{fb}^{-1}$ of integrated luminosity at $\sqrt{s}=14 \mathrm{TeV}$. The red distribution includes all subprocesses of eqs.(2.1)-(2.5), while the blue distribution includes only dominant subprocesses, $q g \rightarrow q g$, of eqs.(2.4)-(2.5). The black histogram shows the SM background.

Note that all these first string excited states are degenerated in mass at tree level, though they have different spins and decay widths.

Two partons in final states are hadronized and give dijet events at the LHC. The dijet invariant mass $M_{j j}$ is an important observable, since a peak or resonance at the mass of string excited states in the $M_{j j}$ distribution may be observed. To make a prediction to the dijet invariant mass distribution, we have to do computer simulations for hadronization and detector simulation. There have been few such works on low-scale string models. We perform Monte Carlo simulations by using CalcHEP [12] for event generation, PYTHIA 8 [13] for hadronization, Delphes 1.9 [14] for detector simulation using its default detector card for ATLAS, and ROOT [15] for analysis of event samples. The details of event generations with string resonances are found in the web page of [16]. In this paper, we do not consider interference effects between the SM processes and the processes with string excited states, because the latter completely dominates at the resonances.

In the rest of paper, we chose a value $M_{\mathrm{s}}=4 \mathrm{TeV}$ as a reference, though the CMS experiment gives a bound $M_{\mathrm{s}}>4 \mathrm{TeV}$ [17]. Fig,2.1 shows the result of simulations of the dijet invariant mass distribution for $M_{\mathrm{s}}=4 \mathrm{TeV}$ and $1.4 \mathrm{fb}^{-1}$ of integrated luminosity at $\sqrt{s}=14 \mathrm{TeV}$. We apply cuts $p_{\mathrm{T}, j_{1}}>330 \mathrm{GeV},\left|y_{1}\right|,\left|y_{2}\right|<2.5$ and $\left|y_{1}-y_{2}\right|<1.3$, where $p_{\mathrm{T}, j_{1}}$ is a largest transverse momentum of jets, and $y_{1}$ and $y_{2}$ are pseudo-rapidities of first and second jets which have primary and secondary large $p_{\mathrm{T}} \mathrm{s}$. We see that contributions from the processes of $q g \rightarrow q g$ are dominant near the resonance at $M_{\mathrm{s}}=4 \mathrm{TeV}$, because of the dominance of quarks in the PDF at high energies. We consider string contributions to $q g \rightarrow q g$ processes only in the following. 


\section{Angular analysis}

The dijet angular distribution exhibits a distinct property in low-scale string models because of spin degeneracy of string excited states. We consider only the process of $q g \rightarrow q g$, because the cross section of the process is dominant near the resonance in the dijet invariant mass distribution. In the process, two first string excited states of quarks with $J=1 / 2$ and $J=3 / 2$ are exchanged in $s$-channel, and they are degenerated in mass. If we can experimentally confirm the degeneracy of states, it is a signature of low-scale string models.

We analyze the $\chi$-distribution on the resonance, which was used by ATLAS experiment to search "new physics" beyond the SM [18] (see Ref.[19] for details). The quality $\chi$ is defined as

$$
\chi=\exp \left(y_{1}-y_{2}\right)=\frac{1+\cos \theta_{*}}{1-\cos \theta_{*}}
$$

where $y_{1}$ and $y_{2}$ are pseudo-rapidities of two jets and $\theta_{*}$ is a scattering angle in the parton centerof-mass frame. For any "new physics" which gives resonances by heavy states, the number of events for small $\chi \sim 1$ is enhanced because of enhancement of scatterings with large angles $\cos \theta_{*} \sim 0$. In the SM, the $\chi$-distribution is flat since $t$ - and $u$-channel exchanges are dominant.

A formula of the $\chi$-distribution is derived in the following way. A cross section of dijet events for a two-parton scattering process is described as

$$
\begin{aligned}
\sigma\left(\mathrm{p}_{1}\left(P_{1}\right), \mathrm{p}_{2}\left(P_{2}\right)\right. & \left.\rightarrow j_{1}\left(p_{1}\right), j_{2}\left(p_{2}\right), X\right) \\
& =\sum_{i} \sum_{j} \int_{0}^{1} d x_{1} \int_{0}^{1} d x_{2} f_{i}\left(x_{1}\right) f_{j}\left(x_{2}\right) \sigma\left(i\left(p_{i}\right), j\left(p_{j}\right) \rightarrow k\left(p_{k}\right), \ell\left(p_{\ell}\right)\right),
\end{aligned}
$$

where $\mathrm{p}_{1}, \mathrm{p}_{2}$ and $X$ denote incoming protons and QCD remnants and $j_{1}, j_{2}$ denote observed jets, and $f_{i}\left(x_{1}\right), f_{j}\left(x_{2}\right)$ are PDFs of protons for initial partons $i, j$ with the momentum fractions

$$
x_{1}=\frac{p_{i}}{P_{1}}, \quad x_{2}=\frac{p_{j}}{P_{2}}
$$

respectively. A cross section of a specific two-parton scattering process $i j \rightarrow k \ell$ can be recast into

$$
\sigma(i j \rightarrow k \ell)=\int_{0}^{1} d x_{1} \int_{0}^{1} d x_{2} f_{i}\left(x_{1}\right) f_{j}\left(x_{2}\right) \int d \hat{t} \frac{d \sigma(i j \rightarrow k \ell)}{d \hat{t}}
$$

where the differential cross section is simply described by a spin-averaged squared amplitude $|\mathcal{M}(i j \rightarrow k \ell)|^{2}$ as

$$
\frac{d \sigma(i j \rightarrow k \ell)}{d \hat{t}}=\frac{|\mathcal{M}(i j \rightarrow k \ell)|^{2}}{16 \pi \hat{s}^{2}}
$$

The formula of the $\chi$-distribution is obtained by performing a change of integrating variables in eq.(3.4).

In the parton center-of-mass frame, rapidities of partons $k$ and $\ell$ are opposite in sign: $y \equiv y_{k}^{*}=-y_{\ell}^{*}$, since these partons are produced back-to-back in this frame. When we define a boost velocity from the parton center-of-mass frame to the proton center-of-mass frame, $\beta \equiv \tanh Y$, pseudo-rapidities of observed jets, $y_{1}$ and $y_{2}$, are written as

$$
y_{1}=y+Y, \quad y_{2}=-y+Y
$$


The quantities

$$
y=\frac{1}{2}\left(y_{1}-y_{2}\right), \quad Y=\frac{1}{2}\left(y_{1}+y_{2}\right),
$$

are independent observables. The necessary kinetic variables in eq.(3.4) are described by $\chi=$ $\exp (2 y), Y$, and the dijet invariant mass $M=\sqrt{\hat{s}}$ as

$$
\begin{gathered}
\hat{t}=-\frac{M^{2}}{1+\chi}, \quad \hat{u}=-\frac{M^{2} \chi}{1+\chi}, \\
x_{1}=\sqrt{\frac{M^{2}}{s}} e^{Y}, \quad x_{2}=\sqrt{\frac{M^{2}}{s}} e^{-Y},
\end{gathered}
$$

where $\sqrt{s}$ is the proton center-of-mass energy. Then we have

$$
\sigma(i j \rightarrow k \ell)=\int d M^{2} \int d Y x_{1} f_{i}\left(x_{1}\right) x_{2} f_{j}\left(x_{2}\right) \int d \chi \frac{1}{(1+\chi)^{2}} \frac{d \sigma(i j \rightarrow k \ell)}{d \hat{t}} .
$$

Inserting the squared amplitude of eqs.(2.4)-(2.5) into eq.(3.10), a prediction to the $\chi$-distribution from low-scale string models is obtained as

$$
\frac{d \sigma(q g \rightarrow q g)}{d \chi}=\frac{1}{(1+\chi)^{2}}\left(C_{1 / 2}+C_{3 / 2} \frac{1+\chi^{3}}{(1+\chi)^{3}},\right)
$$

where $C_{1 / 2}$ and $C_{3 / 2}$ are constants. The term proportional to $C_{1 / 2}$ represents $\chi$-dependence of events with exchanges of $J=1 / 2$ states and the term proportional to $C_{3 / 2}$ represents that of $J=3 / 2$ states. The factor $1 /(1+\chi)^{2}$ in eq.(3.11) is a kinematical factor.

Event samples for the SM only and for the SM with first string excited states in the subprocesses of $q g \rightarrow q g$ are generated. The numbers of generated events are corresponding to $1.8 \mathrm{fb}^{-1}$ and $18.7 \mathrm{fb}^{-1}$ of integrated luminosity at $\sqrt{s}=14 \mathrm{TeV}$. Kinematical cuts imposed in this analysis are

$$
\begin{gathered}
p_{\mathrm{T}, j_{1}}>350 \mathrm{GeV}, \\
M_{\mathrm{s}}-250 \mathrm{GeV}<M_{j j}<M_{\mathrm{s}}+250 \mathrm{GeV}, \\
\left|y_{1}-y_{2}\right|<2.3, \quad\left|y_{1}+y_{2}\right|<2.0 .
\end{gathered}
$$

The same analysis is applied to both event samples for the SM and for the SM with string excited states in the subprocesses of $q g \rightarrow q g$, and we obtain signal event samples by subtracting the first one from the second one.

We fit the $\chi$-distribution of signal events with functions of $\chi$ in eq.(3.11) in three cases of $C_{1 / 2} \neq 0$ and $C_{3 / 2} \neq 0, C_{1 / 2} \neq 0$ and $C_{3 / 2}=0$, and $C_{1 / 2}=0$ and $C_{3 / 2} \neq 0$. These three cases correspond to assumptions with both $J=1 / 2$ and $3 / 2$ states, only a $J=1 / 2$ state and only a $J=3 / 2$ state, respectively. Assuming the state with only $J=1 / 2$, for example, is corresponding to consider new quark-like particles of some other "new physics". The results of these fits are shown in Figs 3.1 3.3.

We clearly see in Fig 3.3 that the fit with only $J=3 / 2$ is not good. The difference of goodness-of-fit between the fit with both $J=1 / 2$ and $3 / 2$ and the one with only $J=1 / 2$, however, are visually not apparent. The $p$-values of these fits are shown in Table 3.1. 


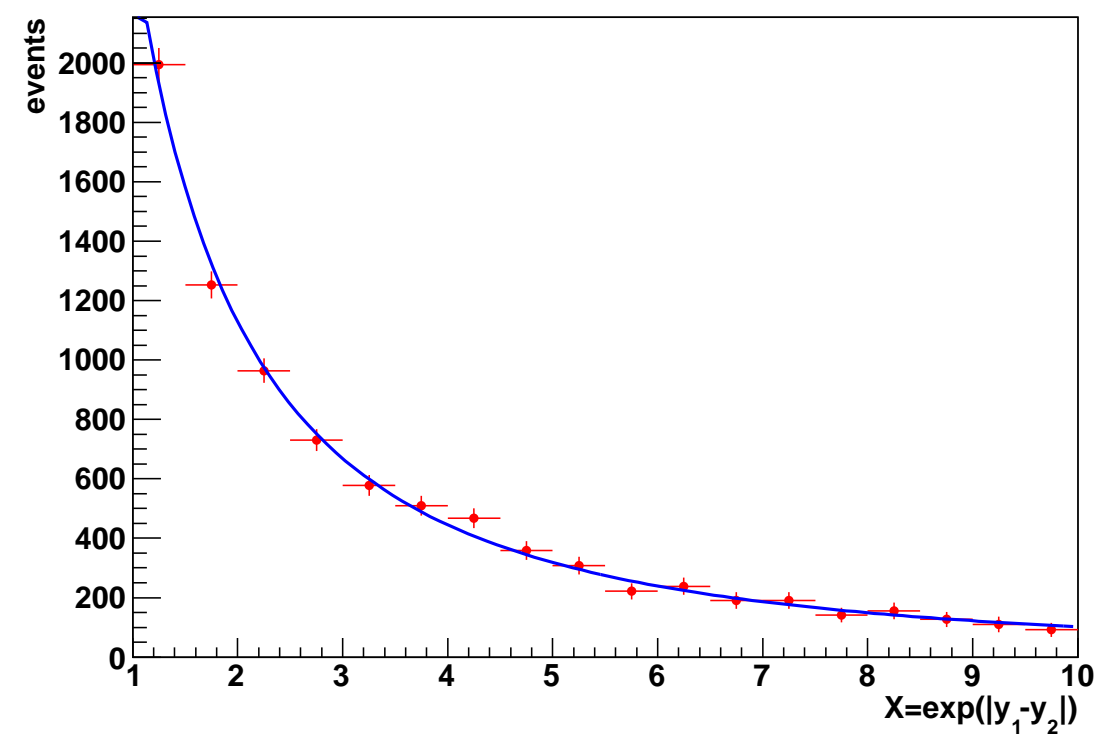

Figure 3.1: The $\chi$-distribution for $M_{\mathrm{s}}=4 \mathrm{TeV}$ with $18.7 \mathrm{fb}^{-1}$ of integrated luminosity at $\sqrt{s}=14 \mathrm{TeV}$. The blue line is a fit with both $J=1 / 2$ and $3 / 2$ states.

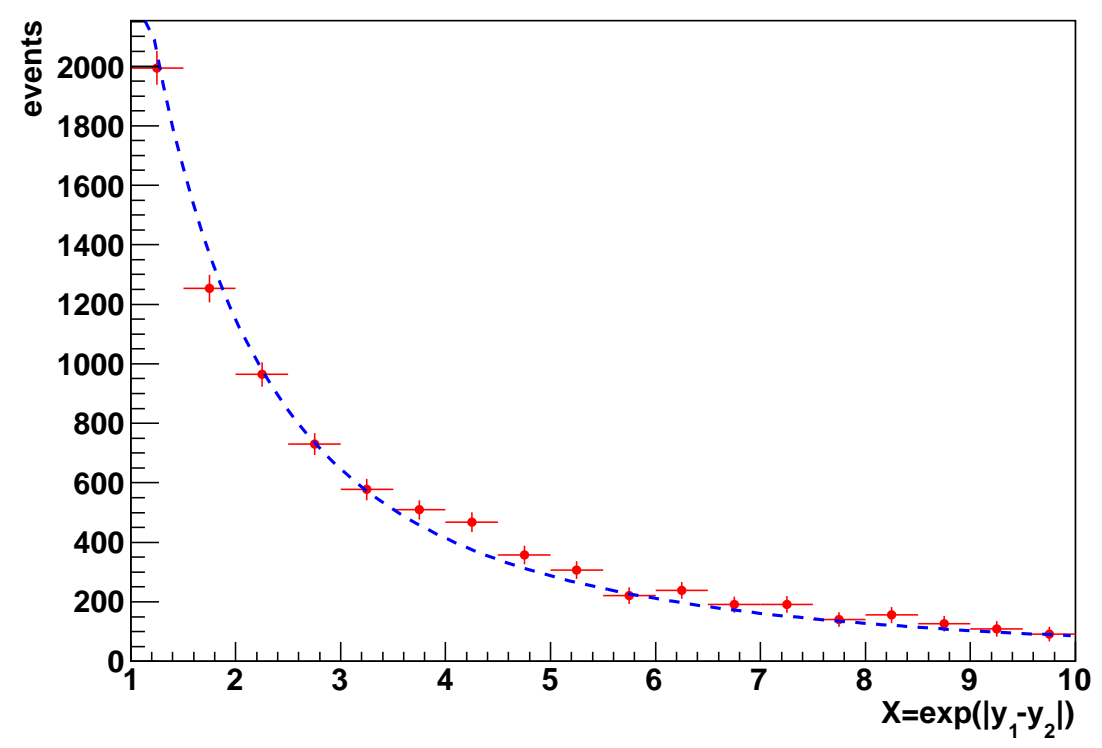

Figure 3.2: The $\chi$-distribution for $M_{\mathrm{s}}=4 \mathrm{TeV}$ with $18.7 \mathrm{fb}^{-1}$ of integrated luminosity at $\sqrt{s}=14 \mathrm{TeV}$. The blue short dashed line is a fit with only a $J=1 / 2$ state. 


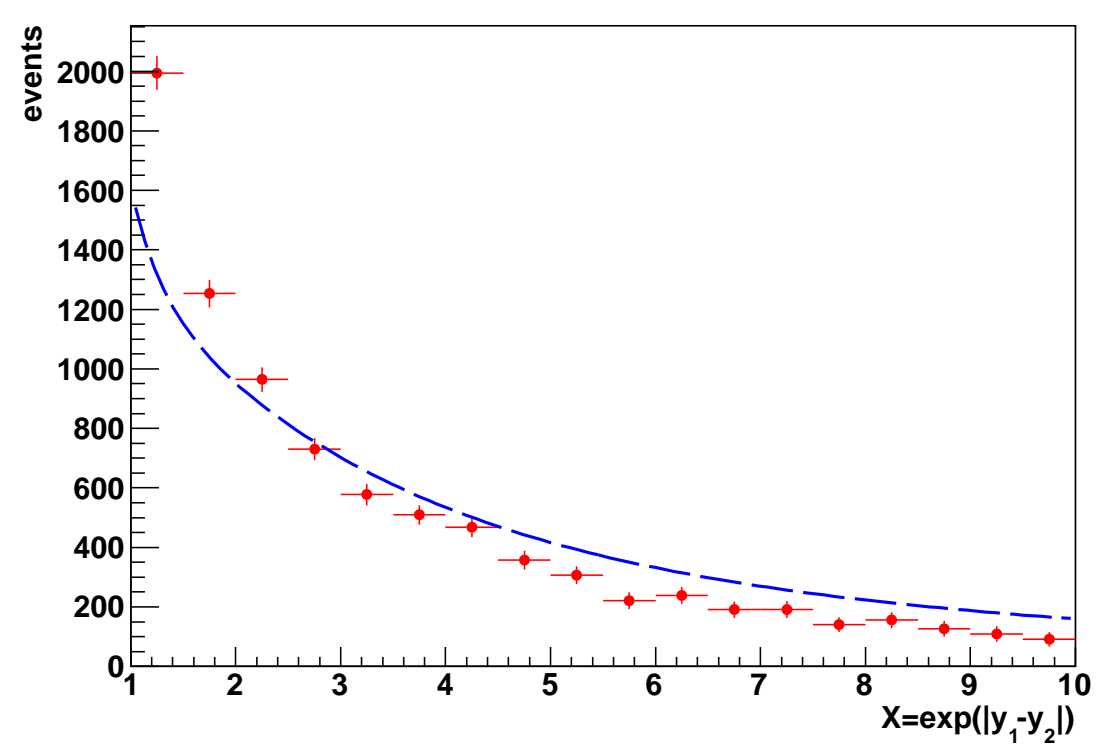

Figure 3.3: The $\chi$-distribution for $M_{\mathrm{s}}=4 \mathrm{TeV}$ with $18.7 \mathrm{fb}^{-1}$ of integrated luminosity at $\sqrt{s}=14 \mathrm{TeV}$. The blue long dashed line is a fit with only a $J=3 / 2$ state.

\begin{tabular}{|c|c|c|c|}
\hline & $J=1 / 2$ and $3 / 2$ & $J=1 / 2$ only & $J=3 / 2$ only \\
\hline $9.8 \mathrm{fb}^{-1}$ & $0.003734(2.20)$ & $0.001187(2.37)$ & $1.248 \times 10^{-31}(11.28)$ \\
$18.7 \mathrm{fb}^{-1}$ & $0.7471(0.747)$ & $0.04058(1.67)$ & $0 .(16.84)$ \\
\hline
\end{tabular}

Table 3.1: $p$-values (reduced $\chi^{2}$ values) of the fits

The $p$-value, in brief, is a probability that a hypothesis is excluded by error in spite of that the hypothesis is correct [20]. Namely, if the $p$-value is small, we can exclude the hypothesis. With $9.8 \mathrm{fb}^{-1}$ of integrated luminosity, $p$-values of the fits with $J=1 / 2$ and $3 / 2$ and with only $J=1 / 2$ are of the same order and very small. It is difficult to distinguish them statistically. However, with $18.7 \mathrm{fb}^{-1}$ of integrated luminosity, the $p$-value of the fit with $J=1 / 2$ and $3 / 2$ is much larger than that of the fit with only $J=1 / 2$. Since a significance level of $p$-values to exclude a hypothesis is usually taken as 0.05 , the fit with $J=1 / 2$ and $3 / 2$ in which the $p$-value is 0.75 much higher than 0.05 can be said to be good, and the fit with only $J=1 / 2$ in which the $p$-value is lower than 0.05 can be excluded. If we look at the $\chi$-distribution of Fig 3.2 closer, we see that the fit in the region $\chi \gtrsim 3.8$ is systematically inconsistent.

We conclude that spin degeneracy at the resonance in the dijet invariant mass distribution by string excited states can be experimentally confirmed. This can be a signature of low-scale string models.

\section{The second string resonances}

Another distinct property of low-scale string models is the appearance of the second resonance in the dijet invariant mass distribution at a specific value of $M_{j j}$. Second string excited 
states have characteristic masses of $M_{2 \text { nd }}=\sqrt{2} M_{\mathrm{s}}$, while second KK modes of the SM particles, for example, have typical masses of $M_{2 \mathrm{nd}}^{\mathrm{KK}} \sim 2 M$ where $M$ is the mass of first KK modes. If a low-scale string model is realized, the second resonance should exist at rather close to the first resonance in the dijet invariant mass distribution.

We calculate spin-averaged squared amplitudes of the dominant processes, $q g \rightarrow q g$ and $q g \rightarrow g q$, with exchanges of second string excited states.

$$
\begin{aligned}
& \left|\mathcal{M}_{2 \text { nd }}(q g \rightarrow q g)\right|^{2}=\left|\mathcal{M}_{2 \text { nd }}(\bar{q} g \rightarrow \bar{q} g)\right|^{2} \\
= & \frac{2\left(N^{2}-1\right)}{N^{2}}\left\{\frac{g^{4}}{2 M_{\mathrm{s}}^{2}}\left[\frac{1}{9} \frac{M_{\mathrm{s}}^{4}(-\hat{u})}{\left(\hat{s}-2 M_{\mathrm{s}}^{2}\right)^{2}+\left(\sqrt{2} M_{\mathrm{s}} \Gamma_{q^{*}, 2 \mathrm{nd}}^{J=1 / 2}\right)^{2}}+\frac{1}{9} \frac{(-\hat{u})(3 \hat{t}+\hat{s})^{2}}{\left(\hat{s}-2 M_{\mathrm{s}}^{2}\right)^{2}+\left(\sqrt{2} M_{\mathrm{s}} \Gamma_{q^{*}, 2 \mathrm{nd}}^{J=3 / 2}\right)^{2}}\right]\right. \\
& \left.+\frac{g^{4}}{8 M_{\mathrm{s}}^{6}}\left[\frac{9}{25} \frac{(-\hat{u})^{3}(5 \hat{t}+\hat{s})^{2}}{\left(\hat{s}-2 M_{\mathrm{s}}^{2}\right)^{2}+\left(\sqrt{2} M_{\mathrm{s}} \Gamma_{q^{*}, 2 \text { nd }}^{J=3 / 2}\right)^{2}}+\frac{1}{25} \frac{M_{\mathrm{s}}^{4}(-\hat{u})^{3}}{\left(\hat{s}-2 M_{\mathrm{s}}^{2}\right)^{2}+\left(\sqrt{2} M_{\mathrm{s}} \Gamma_{q^{*}, 2 \mathrm{nd}}^{J=5 / 2}\right)^{2}}\right]\right\}, \\
= & \frac{2\left(N^{2}-1\right)}{N^{2}}\left\{\frac{g^{4}}{2 M_{\mathrm{s}}^{2}}\left[\frac{1}{9} \frac{M_{\mathrm{s}}^{4}(-\hat{t})}{\left(\hat{s}-2 M_{\mathrm{s}}^{2}\right)^{2}+\left(\sqrt{2} M_{\mathrm{s}} \Gamma_{q^{*}, 2 \mathrm{nd}}^{J=1 / 2}\right)^{2}}+\frac{1}{9} \frac{(-\hat{t})(3 \hat{u}+\hat{s})^{2}}{\left(\hat{s}-2 M_{\mathrm{s}}^{2}\right)^{2}+\left(\sqrt{2} M_{\mathrm{s}} \Gamma_{q^{*}, 2 \mathrm{nd}}^{J=3 / 2}\right)^{2}}\right]\right. \\
& \left.+\frac{g^{4}}{8 M_{\mathrm{s}}^{6}}\left[\frac{9}{25} \frac{(-\hat{t})^{3}(5 \hat{u}+\hat{s})^{2}}{\left(\hat{s}-2 M_{\mathrm{s}}^{2}\right)^{2}+\left(\sqrt{2} M_{\mathrm{s}} \Gamma_{q^{*}, 2 \mathrm{nd}}^{J=3 / 2}\right)^{2}}+\frac{1}{25} \frac{(\hat{s})^{3}}{\left(\hat{s}-2 M_{\mathrm{s}}^{2}\right)^{2}+\left(\sqrt{2} M_{\mathrm{s}} \Gamma_{q^{*}, 2 \mathrm{nd}}^{J=5 / 2}\right)^{2}}\right]\right\} .
\end{aligned}
$$

Total widths of the second excited states of quarks with spin $J, \Gamma_{q^{*}, 2 \text { nd }}^{J}$, are given by

$$
\Gamma_{q^{*}, 2 \text { nd }}^{J=1 / 2}=\frac{g^{2}}{4 \pi} \sqrt{2} M_{\mathrm{s}} \frac{N}{24}, \quad \Gamma_{q^{*}, 2 \text { nd }}^{J=3 / 2}=\frac{g^{2}}{4 \pi} \sqrt{2} M_{\mathrm{s}} \frac{19 N}{240}, \quad \Gamma_{q^{*}, 2 \text { nd }}^{J=5 / 2}=\frac{g^{2}}{4 \pi} \sqrt{2} M_{\mathrm{s}} \frac{N}{60},
$$

where $N=3$ (see Appendix $\mathrm{A}$ ).

We include interference effects between first and second string excited states. The squared amplitudes in eqs.(2.4)-(2.5) and eqs.(4.1)-(4.2) are formulae in a good approximation only around $\hat{s} \simeq M_{\mathrm{s}}^{2}$ and $\hat{s} \simeq 2 M_{\mathrm{s}}^{2}$, respectively. The interference effects should be calculated without replacements of $\hat{s}$ by $M_{\mathrm{s}}^{2}$ or $2 M_{\mathrm{s}}^{2}$ in the processes of deriving eqs.(2.4)-(2.5) and eqs.(4.1)-(4.2), respectively (see Appendix $\mathrm{A}$ for details).

The structure of the amplitudes for the processes of $q g \rightarrow q g$ is obtained as

$$
A_{q g \rightarrow q g}=A_{1 \mathrm{st}}^{J=1 / 2}+A_{2 \mathrm{nd}}^{J=1 / 2}+A_{2 \mathrm{nd}}^{J=3 / 2},
$$

where $A_{n \text {th }}^{J}$ describe contributions of $n$th string excited states with spin $J$. The squared amplitude includes three kinds of interference terms.

$$
\begin{aligned}
\left|A_{q g \rightarrow q g}\right|^{2} & =\left|A_{1 \mathrm{st}}^{J=1 / 2}\right|^{2}+\left|A_{2 \text { nd }}^{J=1 / 2}\right|^{2}+\left|A_{2 \text { nd }}^{J=3 / 2}\right|^{2} \\
& +\left(A_{1 \mathrm{st}}^{J=1 / 2} A_{2 \text { nd }}^{J=1 / 2^{*}}+\text { c.c }\right)+\left(\mathrm{A}_{1 \mathrm{st}}^{\mathrm{J}=1 / 2} \mathrm{~A}_{2 \mathrm{nd}}^{\mathrm{J}=3 / 2^{*}}+\text { c.c }\right)+\left(\mathrm{A}_{2 \mathrm{nd}}^{\mathrm{J}=1 / 2} \mathrm{~A}_{2 \mathrm{nd}}^{\mathrm{J}=3 / 2^{*}}+\text { c.c }\right) .
\end{aligned}
$$

The last term, an interference between two amplitudes with poles at the same place, $M_{2 \mathrm{nd}}^{2}=$ $2 M_{\mathrm{s}}^{2}$, gives large contributions to the total peak cross section at $\hat{s}=2 M_{\mathrm{s}}^{2}$. The other two interference terms give small suppression effects in the region of $M_{\mathrm{s}}^{2}<\hat{s}<2 M_{\mathrm{s}}^{2}$. The interference 


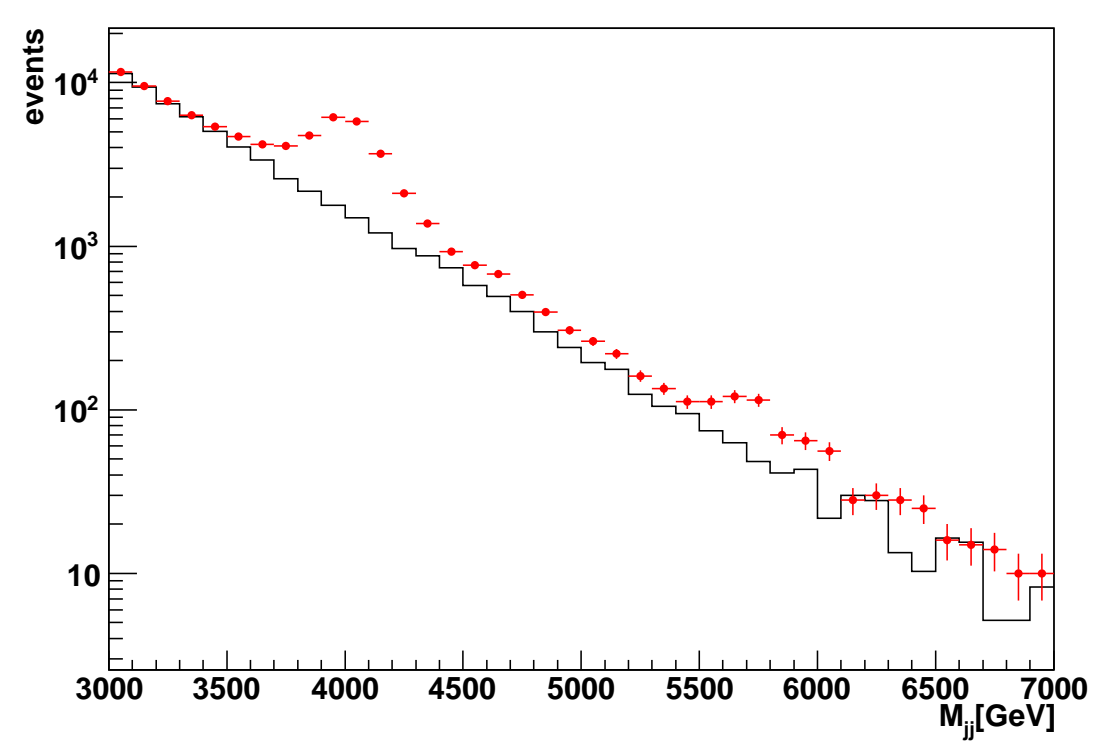

Figure 4.1: The dijet invariant mass distribution with interference effects between first and second excited states for $M_{\mathrm{s}}=4 \mathrm{TeV}$ with $50 \mathrm{fb}^{-1}$ of integrated luminosity at $\sqrt{s}=14 \mathrm{TeV}$. Black histogram shows the SM background.

effects between the SM contributions and string contributions are not included. There should be some effects due to $\hat{u}$ - and $\hat{t}$-channel exchanges of string excited states, which might give a certain effect in the region of $M_{\mathrm{s}}^{2}<\hat{s}<2 M_{\mathrm{s}}^{2}$. We leave detailed analyses in these effects for future works. Without including these effects, the peak cross sections at $\hat{s}=M_{\mathrm{s}}$ and $\hat{s}=2 M_{\mathrm{s}}^{2}$ should be correctly estimated, which is sufficient for the aim of this paper.

Event samples for the SM with first and second string excited states are generated. The number of events is corresponding to $50 \mathrm{fb}^{-1}$ at $\sqrt{s}=14 \mathrm{TeV}$. Kinematical cuts imposed in this analysis are

$$
\begin{gathered}
p_{\mathrm{T}, j_{1}}>350 \mathrm{GeV} \\
\left|y_{1,2}\right|<2.3, \quad\left|y_{1}-y_{2}\right|<1.7 .
\end{gathered}
$$

The dijet invariant mass distribution is shown in Fig 4.1. We see the first string resonance at $M_{j j}=M_{\mathrm{s}}=4 \mathrm{TeV}$ and the second string resonance at $M_{j j}=\sqrt{2} \times M_{\mathrm{s}} \simeq 5.66 \mathrm{TeV}$. We need integrated luminosity of $50 \mathrm{fb}^{-1}$ to obtain enough events at high energies and to see the second string resonance clearly. Signal-to-noise ratios for the second string resonance are calculated in the dijet invariant mass window $\left[\sqrt{2} M_{\mathrm{s}}-250 \mathrm{GeV}, \sqrt{2} M_{\mathrm{s}}+250 \mathrm{GeV}\right]$. For $18.5 \mathrm{fb}^{-1}$ of integrated luminosity, $S / \sqrt{B}=71 / \sqrt{133} \simeq 6 \sigma$, and for $50 \mathrm{fb}^{-1}, S / \sqrt{B}=217 / \sqrt{313} \simeq 12 \sigma$.

If a resonance in the dijet invariant mass distribution is observed at the LHC, we should look for a second resonance at rather close to the first resonance. If the second resonance is discovered at a place $\sqrt{2}$ times far from the place of the first resonance, which is a strong signature for low-scale string models. 


\section{Conclusions}

We have investigated phenomenology of low-scale string models at the LHC. Since in lowscale string models, the string scale $M_{s}$ is of the order of $\mathrm{TeV}$ with large extra dimensions, it is expected some string excited states as well as KK states may be observed at the LHC. It has been known that parton two-body scattering processes at the LHC is highly model independent, namely independent from the way of compactification of extra dimensions, and processes with single KK state in $s$-channel are highly suppressed. Therefore, dijet events at the LHC are that should be focused to observe string excited states.

The string excited states appear as resonances in the dijet invariant mass distribution. Since several string excited states with different spins are degenerated in mass of $M_{s}$, dijet events on the resonance exhibit special angular distributions which are not realized in any other "new physics" with a single heavy state. We have investigated the $\chi$-distribution on the resonance by Monte Carlo simulations and shown that main contributions of two kinds of string excited states of quarks with spin $J=1 / 2$ and $J=3 / 2$ can be confirmed with $20 \mathrm{fb}^{-1}$ of integrated luminosity at $\sqrt{s}=14 \mathrm{TeV}$.

Another inevitable prediction of low-scale string models is emergence of a second resonance in the dijet invariant mass distribution due to second string excited states of quarks and gluons. A distinct property is the place of the second resonance, namely the mass of second string

excited states. The masses should be $\sqrt{2} M_{s}$ which should be compared with that masses of second KK states is twice that of first KK states. We have shown by Monte Carlo simulations that the second resonance in the dijet invariant mass distribution due to second string excited states can be observed with $50 \mathrm{fb}^{-1}$ of integrated luminosity at $\sqrt{s}=14 \mathrm{TeV}$.

Though these are necessary and inevitable signatures of low-scale string models, more information is necessary to establish low-scale string. For example, confirmation of special angular distributions of dijet events on the second resonance, and emergence of light anomalous U(1) gauge bosons with special interactions related with anomaly structures (though this is rather highly model dependent) should be worth to investigate in future. We are planning a systematic and detailed study of second string excited states with larger values of string scales than $4 \mathrm{TeV}$ which is the current lower bound by the CMS experiment.

Note added. After accepting this article for publication, we became aware that the importance of second excited states has been pointed out in ref. [21].

\section{Acknowledgements}

The authors would like to thank Koji Terashi for valuable advices on the experiment of dijet physics and techniques of Monte Carlo simulations. The authors would like to thank Junpei Maeda for advices on the techniques of Monte Carlo simulations. N.K. is supported in part by Grand-in-Aid for Research No. DC203 from Tokyo Metropolitan University. 


\section{A Amplitudes and widths with string excited states}

Open-string two-body scattering amplitudes between quarks and gluons are calculated by the world-sheet superconformal field theory in flat space-time as

$$
\begin{aligned}
& \mathcal{M}\left(q_{1}^{ \pm} g_{2}^{ \pm} \rightarrow q_{3}^{ \pm} g_{4}^{ \pm}\right)=2 g^{2} \sqrt{-\frac{s}{u}}\left[\frac{s}{t} V_{s}\left(T^{a_{2}} T^{a_{4}}\right)_{\alpha_{3} \alpha_{1}}+\frac{u}{t} V_{u}\left(T^{a_{4}} T^{a_{2}}\right)_{\alpha_{3} \alpha_{1}}\right], \\
& \mathcal{M}\left(q_{1}^{ \pm} g_{2}^{\mp} \rightarrow q_{3}^{ \pm} g_{4}^{\mp}\right)=2 g^{2} \sqrt{-\frac{u}{s}}\left[\frac{s}{t} V_{s}\left(T^{a_{2}} T^{a_{4}}\right)_{\alpha_{3} \alpha_{1}}+\frac{u}{t} V_{u}\left(T^{a_{4}} T^{a_{2}}\right)_{\alpha_{3} \alpha_{1}}\right],
\end{aligned}
$$

where $\alpha_{1}, \alpha_{3}$ are color indices of quarks, $a_{2}, a_{4}$ are color indices of gluons, and $s, t$ and $u$ are the Mandelstam variables of partons with $s+t+u=0$. Here, the generators $T^{a_{i}}$ are for the fundamental representation of $\mathrm{SU}(3)_{\text {color }}$ gauge group. Amplitudes $\mathcal{M}\left(q_{1}^{ \pm} g_{2}^{ \pm} \rightarrow g_{3}^{ \pm} q_{4}^{ \pm}\right)$and $\mathcal{M}\left(q_{1}^{ \pm} g_{2}^{\mp} \rightarrow g_{3}^{\mp} q_{4}^{ \pm}\right)$are obtained by replacements of $u \leftrightarrow t$ and $3 \leftrightarrow 4$ in the above amplitudes of eqs.(A.1)-(A.2).

The functions $V_{s, t, u}$ in eqs.(A.1)-(A.2) are "form factor" functions of the Mandelstam variables which represent string effects in open-string amplitudes.

$$
V_{t}=V(s, t, u), \quad V_{u}=V(t, u, s), \quad V_{s}=V(u, s, t)
$$

where

$$
V(s, t, u)=\frac{\Gamma\left(1-s / M_{\mathrm{s}}^{2}\right) \Gamma\left(1-u / M_{\mathrm{s}}^{2}\right)}{\Gamma\left(1+t / M_{\mathrm{s}}^{2}\right)}=\frac{1}{M_{\mathrm{s}}^{2}} \frac{s u}{t} \frac{\Gamma\left(-s / M_{\mathrm{s}}^{2}\right) \Gamma\left(-u / M_{\mathrm{s}}^{2}\right)}{\Gamma\left(t / M_{\mathrm{s}}^{2}\right)} .
$$

These functions are expanded by sums over infinite $s$-channel poles.

$$
V_{t}=\frac{1}{M_{\mathrm{s}}^{2}} \frac{s u}{t}\left\{-\sum_{n=1}^{\infty} \frac{1}{n !} \frac{1}{\left(M_{\mathrm{s}}^{2}\right)^{n-1}} \frac{1}{s-n M_{\mathrm{s}}^{2}} \prod_{J=1}^{n}\left(u+J M_{\mathrm{s}}^{2}\right)+\mathcal{O}\left(\left(s-n M_{\mathrm{s}}^{2}\right)^{0}\right)\right\} .
$$

Note that the function $V_{s}$ has no $s$-channel poles. The terms of $\mathcal{O}\left(\left(s-n M_{\mathrm{s}}^{2}\right)^{0}\right)$ in eq. A.5 can be neglected if we consider only near each $n$th pole: $s \simeq n M_{\mathrm{s}}^{2}$. The each pole corresponds to $n$th string excited state with mass $M_{n}=\sqrt{n} M_{\mathrm{s}}$.

The form factor functions are also expanded by $\left(M_{\mathrm{s}}^{2}\right)^{-1}$ as

$$
V_{t}=1-\frac{\pi^{2}}{6} s u\left(\frac{1}{M_{\mathrm{s}}^{2}}\right)^{2}+\mathcal{O}\left(\left(M_{\mathrm{s}}^{2}\right)^{-3}\right)
$$

which corresponds to the low-energy limit, namely $V_{s, t, u} \rightarrow 1$ with $M_{\mathrm{s}} \rightarrow \infty$. In a low-energy limit, the open-string amplitudes of $q g \rightarrow q g$ in eqs.(A.1)-(A.2) exactly coincide with the QCD amplitudes,

$$
\begin{aligned}
& \mathcal{M}_{\mathrm{QCD}}\left(q_{1}^{ \pm} g_{2}^{ \pm} \rightarrow q_{3}^{ \pm} g_{4}^{ \pm}\right)=2 g^{2} \sqrt{-\frac{s}{u}}\left[\frac{s}{t}\left(T^{a_{2}} T^{a_{4}}\right)_{\alpha_{3} \alpha_{1}}+\frac{u}{t}\left(T^{a_{4}} T^{a_{2}}\right)_{\alpha_{3} \alpha_{1}}\right] \\
& \mathcal{M}_{\mathrm{QCD}}\left(q_{1}^{ \pm} g_{2}^{\mp} \rightarrow q_{3}^{ \pm} g_{4}^{\mp}\right)=2 g^{2} \sqrt{-\frac{u}{s}}\left[\frac{s}{t}\left(T^{a_{2}} T^{a_{4}}\right)_{\alpha_{3} \alpha_{1}}+\frac{u}{t}\left(T^{a_{4}} T^{a_{2}}\right)_{\alpha_{3} \alpha_{1}}\right]
\end{aligned}
$$




\section{A.1 The first excited states}

In case of $n=1$, the form factor functions are approximated near the first $s$-channel pole, $s \simeq M_{\mathrm{s}}^{2}$, as

$$
V_{t} \simeq \frac{u}{s-M_{\mathrm{s}}^{2}}, \quad V_{u} \simeq \frac{t}{s-M_{\mathrm{s}}^{2}} .
$$

Amplitudes of $q g \rightarrow q g$ with exchanges of the first quark excited states in eqs.(A.1)-(A.2) are recast into

$$
\begin{aligned}
& \mathcal{M}_{1 \mathrm{st}}\left(q_{1}^{ \pm} g_{2}^{ \pm} \rightarrow q_{3}^{ \pm} g_{4}^{ \pm}\right) \simeq-2 g^{2} M_{\mathrm{s}}^{2} \frac{1}{s-M_{\mathrm{s}}^{2}} d_{\mp 1 / 2, \mp 1 / 2}^{J=1 / 2}(\theta)\left(T^{a_{4}} T^{a_{2}}\right)_{\alpha_{3} \alpha_{1}}, \\
& \mathcal{M}_{1 \mathrm{st}}\left(q_{1}^{ \pm} g_{2}^{\mp} \rightarrow q_{3}^{ \pm} g_{4}^{\mp}\right) \simeq-2 g^{2} M_{\mathrm{s}}^{2} \frac{1}{s-M_{\mathrm{s}}^{2}} d_{ \pm 3 / 2, \pm 3 / 2}^{J=3 / 2}(\theta)\left(T^{a_{4}} T^{a_{2}}\right)_{\alpha_{3} \alpha_{1}}
\end{aligned}
$$

where $\mathrm{d}_{J_{z}, J_{z}^{\prime}}^{J}(\theta)$ s are the Wigner $d$-functions,

$$
d_{ \pm 1 / 2, \pm 1 / 2}^{J=1 / 2}(\theta)=\cos \frac{\theta}{2}, \quad d_{ \pm 3 / 2, \pm 3 / 2}^{J=3 / 2}(\theta)=\cos \frac{\theta}{2}\left(\frac{1+\cos \theta}{2}\right)
$$

The Wigner $d$-function $d_{J_{z}, J_{z}^{\prime}}^{J}(\theta)$ represents angular dependence of a state with spin $J$ with conditions of the initial spin component $J_{z}$ along the $z$-axis and the final spin component $J_{z}^{\prime}$ along the $z^{\prime}$-axis. The angle $\theta$ is that between the $z$-axis and $z^{\prime}$-axis. Therefore, the exchanged state in the process of eq. (A.10) with $J_{z}= \pm 1 / 2$ are first quark excited states with $J=1 / 2$ and the exchanged states in the process of eq. A.11) with $J_{z}= \pm 3 / 2$ are first quark excited state with $J=3 / 2$.

The $s$-channel poles in eq. (A.5) have to be softened into Breit-Wigner forms:

$$
\frac{1}{s-n M_{\mathrm{s}}^{2}} \rightarrow \frac{1}{s-n M_{\mathrm{s}}^{2}+i \sqrt{n} M_{\mathrm{s}} \Gamma_{q^{*}, n \mathrm{th}}^{J}},
$$

where the widths of the $n$th excited states of quarks $q^{*}$ with spin $J, \Gamma_{q^{*}, n \text {th }}^{J}$, are added by hand.

The width is calculated as follows. A Lorentz invariant amplitude in a two-body scattering process, in which a state with mass $M$, spin $J$ and the gauge index $\alpha$ is exchanged in $s$-channel, is written in the following form,

$$
\mathcal{M}=-\sum_{\alpha, J} \frac{1}{s-M^{2}} F_{\lambda_{3}, \lambda_{4} ; \alpha_{3}, a_{4}}^{\alpha J} F_{\lambda_{1}, \lambda_{2} ; \alpha_{1}, a_{2}}^{\alpha J} d_{\lambda_{1}-\lambda_{2}, \lambda_{3}-\lambda_{4}}^{J}(\theta),
$$

where the entire negative sign is just a convention. The quantities $F_{\lambda_{i}, \lambda_{j} ; \alpha_{i}, a_{j}}^{\alpha J}$ are vertex factors in decay of the exchanged state in the process of eq.(A.14) with spin $J$ and the gauge index $\alpha$ into two states with the helicities $\lambda_{i}, \lambda_{j}$ and gauge indices $\alpha_{i}, a_{j}$, which are independent from a spin component $J_{z}$ of the exchanged state. The decay width of the exchanged state can be calculated as

$$
\Gamma_{\lambda_{3}, \lambda_{4} ; \alpha_{3}, a_{4}}^{\alpha J}=\frac{1}{16 \pi M} \frac{1}{2 J+1}\left|F_{\lambda_{3}, \lambda_{4} ; \alpha_{3}, a_{4}}^{\alpha J}\right|^{2}
$$

See Ref.[11] for details. 
From the amplitudes of eqs.(A.10)-(A.11), we have

$$
\left.F_{ \pm 1 / 2, \pm 1 ; \alpha_{3}, a_{4}}^{\alpha J=1 / 2}\right|_{1 \text { st }}=\sqrt{2} g M_{\mathrm{s}}\left(T^{a_{4}}\right)_{\alpha \alpha_{3}},\left.\quad F_{ \pm 1 / 2, \mp 1 ; \alpha_{3}, a_{4}}^{\alpha J=3 / 2}\right|_{1 \mathrm{st}}=\sqrt{2} g M_{\mathrm{s}}\left(T^{a_{4}}\right)_{\alpha \alpha_{3}} .
$$

Color-averaged decay widths of the first quark excited state $q^{*}$ with $J$ in a decay process of $q^{*} \rightarrow q G$ are calculated as

$$
\Gamma_{q^{*} \rightarrow q G, 1 \mathrm{st}}^{J}=\frac{1}{16 \pi M_{\mathrm{s}}} \frac{1}{2 J+1} \frac{1}{N} \sum_{\alpha} \sum_{\alpha_{3}, a_{4}} \sum_{\lambda_{3}, \lambda_{4}}\left|F_{\lambda_{3}, \lambda_{4} ; \alpha_{3}, a_{4}}^{\alpha J}\right|^{2},
$$

where $N=3$. In case of $J=1 / 2$,

$$
\begin{aligned}
\Gamma_{q^{*} \rightarrow q G, 1 \mathrm{st}}^{J=1 / 2} & =\frac{1}{16 \pi M_{\mathrm{s}}} \frac{1}{2 \times \frac{1}{2}+1} \frac{1}{N} \sum_{\alpha} \sum_{\alpha_{3}, a_{4}} \frac{1}{2}\left\{\left|F_{+1 / 2,+1 ; \alpha_{3}, a_{4}}^{\alpha J=1 / 2}\right|^{2}+\left|F_{-1 / 2,-1 ; \alpha_{3}, a_{4}}^{\alpha J=1 / 2}\right|^{2}\right\} \\
& =\frac{g^{2} M_{\mathrm{s}}}{16 \pi} \frac{1}{N} \sum_{a_{4}} \operatorname{tr}\left(T^{a_{4}} T^{a_{4}}\right) .
\end{aligned}
$$

The factor $\frac{1}{2}$ of a sum over helicities comes from a fact that the final state helicity configuration $\left(\lambda_{3}, \lambda_{4}\right)=(+1 / 2,+1)$ couples only to the initial state helicity $J_{z}=-1 / 2$, while $\left(\lambda_{3}, \lambda_{4}\right)=$ $(-1 / 2,-1)$ couples only to $J_{z}=+1 / 2$. The same applies to the case of $J=3 / 2$ (see eqs.(A.10)(A.11)).

The decay products $G$ in the process of $q^{*} \rightarrow q G$ are gauge bosons of $\mathrm{U}(3)_{\text {color }}$ gauge group. The $\mathrm{U}(N)$ gauge bosons $G^{A}\left(A=1, \cdots, N^{2}\right)$ are split into $\mathrm{SU}(N)$ gauge bosons $g^{a}$ ( $\left.a=1, \cdots, N^{2}-1\right)$, namely gluons in case of $N=3$ and a $\mathrm{U}(1)$ gauge boson $C^{0}$. The $\mathrm{U}(N)$ generators $T^{A}$ are also split into $\mathrm{SU}(N)$ generators $T^{a}$ with $\operatorname{tr}\left(T^{a} T^{b}\right)=\frac{1}{2} \delta^{a b}$ and a $\mathrm{U}(1)$ generator $T^{0}=\frac{1}{\sqrt{2 N}} \mathbf{1}_{N}$. Hence,

$$
\begin{array}{rlrl}
\frac{1}{N} \sum_{a=1}^{N^{2}-1} \operatorname{tr}\left(T^{a} T^{a}\right) & =\frac{N^{2}-1}{2 N} & & \text { for } q^{*} \rightarrow q g, \\
\frac{1}{N} \operatorname{tr}\left(T^{0} T^{0}\right) & =\frac{1}{2 N} & \text { for } q^{*} \rightarrow q C^{0} .
\end{array}
$$

Total widths of the first quark excited states with $J=1 / 2$ and $J=3 / 2$ are obtained as eq.(2.8).

Spin- and color-averaged squared amplitude of $q g \rightarrow q g$ with exchanges of the first quark excited states with $J=1 / 2$ and $J=3 / 2$ is calculated from eqs.(A.10)-(A.11) as

$$
\begin{aligned}
&\left|\mathcal{M}_{1 \mathrm{st}}\left(q_{1} g_{2} \rightarrow q_{3} g_{4}\right)\right|^{2}= \frac{1}{N} \frac{1}{N^{2}-1} \sum_{\alpha_{1}, a_{2}} \sum_{\alpha_{3}, a_{4}} \\
& \times\left(\frac{1}{2}\right)^{2}\left\{\left|\mathcal{M}_{1 \mathrm{st}}\left(q_{1}^{+} g_{2}^{+} \rightarrow q_{3}^{+} g_{4}^{+}\right)\right|^{2}+\left|\mathcal{M}_{1 \mathrm{st}}\left(q_{1}^{-} g_{2}^{-} \rightarrow q_{3}^{-} g_{4}^{-}\right)\right|^{2}\right. \\
&\left.\quad+\left|\mathcal{M}_{1 \mathrm{st}}\left(q_{1}^{+} g_{2}^{-} \rightarrow q_{3}^{+} g_{4}^{-}\right)\right|^{2}+\left|\mathcal{M}_{1 \mathrm{st}}\left(q_{1}^{-} g_{2}^{+} \rightarrow q_{3}^{-} g_{4}^{+}\right)\right|^{2}\right\} .
\end{aligned}
$$

This is equal to the right-handed side of the second line of eq.(2.4). 


\section{A.2 The second excited states}

In case of $n=2$, the form factor functions are approximated near the second $s$-channel pole, $s \simeq 2 M_{\mathrm{s}}^{2}$, as

$$
V_{t} \simeq \frac{1}{M_{\mathrm{s}}^{2}} \frac{u}{s-2 M_{\mathrm{s}}^{2}}\left(u+\frac{s}{2}\right), \quad V_{t} \simeq \frac{1}{M_{\mathrm{s}}^{2}} \frac{t}{s-2 M_{\mathrm{s}}^{2}}\left(t+\frac{s}{2}\right) .
$$

Amplitudes of $q g \rightarrow q g$ with exchanges of the second quark excited states are recast into

$$
\begin{aligned}
& \mathcal{M}_{2 \text { nd }}\left(q_{1}^{ \pm} g_{2}^{ \pm} \rightarrow q_{3}^{ \pm} g_{4}^{ \pm}\right) \simeq-4 g^{2} M_{\mathrm{s}}^{2} \frac{1}{s-2 M_{\mathrm{s}}^{2}}\left[\frac{1}{3} d_{\mp 1 / 2, \mp 1 / 2}^{J=1 / 2}(\theta)+\frac{2}{3} d_{\mp 1 / 2, \mp 1 / 2}^{J=3 / 2}(\theta)\right]\left(T^{a_{4}} T^{a_{2}}\right)_{\alpha_{3} \alpha_{1}} \\
& \mathcal{M}_{2 \text { nd }}\left(q_{1}^{ \pm} g_{2}^{\mp} \rightarrow q_{3}^{ \pm} g_{4}^{\mp}\right) \simeq-4 g^{2} M_{\mathrm{s}}^{2} \frac{1}{s-2 M_{\mathrm{s}}^{2}}\left[\frac{3}{5} d_{ \pm 3 / 2, \pm 3 / 2}^{J=3 / 2}(\theta)+\frac{2}{5} d_{ \pm 3 / 2, \pm 3 / 2}^{J=5 / 2}(\theta)\right]\left(T^{a_{4}} T^{a_{2}}\right)_{\alpha_{3} \alpha_{1}}
\end{aligned}
$$

where the Wigner $d$-functions are given in eq.(A.12) and

$$
d_{ \pm 1 / 2, \pm 1 / 2}^{J=3 / 2}(\theta)=\cos \frac{\theta}{2}\left(\frac{3 \cos \theta-1}{2}\right), \quad d_{ \pm 3 / 2, \pm 3 / 2}^{J=5 / 2}(\theta)=\cos \frac{\theta}{2}\left(\frac{1+\cos \theta}{2}\right)\left(\frac{5 \cos \theta-3}{2}\right) .
$$

Therefore, the exchanged states in the process of eq. (A.22) with $J_{z}= \pm 1 / 2$ are second quark excited states with $J=1 / 2$ and $J=3 / 2$, and the exchanged states in the process of eq. (A.23) with $J_{z}= \pm 3 / 2$ are second quark excited states with $J=3 / 2$ and $J=5 / 2$.

From the amplitudes of eqs. A.22)-(A.23), we have

$$
\begin{array}{c|c|c|c|c|}
\left.F_{ \pm 1 / 2, \pm 1 ; \alpha_{3}, a_{4}}^{\alpha J=1 / 2}\right|_{2 \text { nd }}=\frac{2}{\sqrt{3}} g M_{\mathrm{s}}\left(T^{a_{4}}\right)_{\alpha \alpha_{3}}, & \left.F_{ \pm 1 / 2, \pm 1 ; \alpha_{3}, a_{4}}^{\alpha J=3 / 2}\right|_{2 \text { nd }}=2 \sqrt{\frac{2}{3}} g M_{\mathrm{s}}\left(T^{a_{4}}\right)_{\alpha \alpha_{3}}, \\
\left.F_{ \pm 1 / 2, \mp 1 ; \alpha_{3}, a_{4}}^{\alpha J=3 / 2}\right|_{2 \text { nd }}=2 \sqrt{\frac{3}{5}} g M_{\mathrm{s}}\left(T^{a_{4}}\right)_{\alpha \alpha_{3}}, & \left.F_{ \pm 1 / 2, \mp 1 ; \alpha_{3}, a_{4}}^{\alpha J=5 / 2}\right|_{2 \text { nd }}=2 \sqrt{\frac{2}{5}} g M_{\mathrm{s}}\left(T^{a_{4}}\right)_{\alpha \alpha_{3}} .
\end{array}
$$

Color-averaged decay widths of the second quark excited state $q^{*}$ with $J$ are calculated. In case of $J=3 / 2$, since there are contributions from both processes of eq.(A.22) and eq.(A.23) with exchanges of states with $J_{z}= \pm 1 / 2$ and $J_{z}= \pm 3 / 2$, respectively,

$$
\begin{aligned}
\Gamma_{q^{*}, 2 \mathrm{nd}}^{J=3 / 2}= & \frac{1}{16 \pi \sqrt{2} M_{\mathrm{s}}} \frac{1}{2 \times \frac{3}{2}+1} \frac{1}{N} \sum_{\alpha} \sum_{\alpha_{3}, a_{4}} \frac{1}{2}\left\{\left|F_{+1 / 2,+1 ; \alpha_{3}, a_{4}}^{\alpha J=3 / 2}\right|^{2}+\left|F_{-1 / 2,-1 ; \alpha_{3}, a_{4}}^{\alpha J=3 / 2}\right|^{2}\right. \\
& \left.+\left|F_{+1 / 2,-1 ; \alpha_{3}, a_{4}}^{\alpha J=3 / 2}\right|^{2}+\left|F_{-1 / 2,+1 ; \alpha_{3}, a_{4}}^{\alpha J=3 / 2}\right|^{2}\right\} \\
= & \frac{g^{2} M_{\mathrm{s}}}{16 \sqrt{2} \pi}\left(\frac{2}{3}+\frac{3}{5}\right) \frac{1}{N} \sum_{a_{4}} \operatorname{tr}\left(T^{a_{4}} T^{a_{4}}\right) .
\end{aligned}
$$

Total widths of the second quark excited states with $J=1 / 2, J=3 / 2$ and $J=5 / 2$ are obtained as eq.(4.3). We are neglecting subdominant decay processes to the first string excited states.

The spin- and color-averaged squared amplitude is calculated from eqs.(A.22)-(A.23) in a similar way to eq.(A.20) and the result is equal to eq.(4.1). 


\section{A.3 The interference between the first and second excited states}

The interference effects between first and second excited states have to be calculated without replacements of $s$ with $M_{\mathrm{s}}^{2}$ in eqs.(A.10)-(A.11) and with $2 M_{\mathrm{s}}^{2}$ in eqs.(A.22)-(A.23). It is because we need amplitudes in the region of $s$ a little far from the poles of first and second excited states.

Amplitudes of $q g \rightarrow q g$ with exchanges of the first and second quark excited sates are written as

$$
\begin{aligned}
& \mathcal{M}_{1 \mathrm{st}+2 \mathrm{nd}}\left(q_{1}^{ \pm} g_{2}^{ \pm} \rightarrow q_{3}^{ \pm} g_{4}^{ \pm}\right)=2 g^{2}\left(T^{a_{4}} T^{a_{2}}\right)_{\alpha_{3} \alpha_{1}} \\
& \times\left\{-\frac{s^{2}}{M_{\mathrm{s}}^{2}} \sqrt{-\frac{u}{s}} \frac{1}{s-M_{\mathrm{s}}^{2}+i M_{\mathrm{s}} \Gamma_{q^{*}, 1 \mathrm{st}}^{J=1 / 2}}\right. \\
& \left.\quad-\frac{s^{3}}{4 M_{\mathrm{s}}^{4}}\left[\frac{1}{3} \sqrt{-\frac{u}{s}} \frac{1}{s-2 M_{\mathrm{s}}^{2}+i \sqrt{2} M_{\mathrm{s}} \Gamma_{q^{*}, 2 \mathrm{nd}}^{J=1 / 2}}+\frac{2}{3} \sqrt{-\frac{u}{s}}\left(\frac{3 t+s}{s}\right) \frac{1}{s-2 M_{\mathrm{s}}^{2}+i \sqrt{2} M_{\mathrm{s}} \Gamma_{q^{*}, 2 \mathrm{nd}}^{J=3 / 2}}\right]\right\}, \quad \text { (A.28) } \\
& \mathcal{M}_{1 \mathrm{st}+2 \mathrm{nd}\left(q_{1}^{ \pm} g_{2}^{\mp} \rightarrow q_{3}^{ \pm} g_{4}^{\mp}\right)=2 g^{2}\left(T^{a_{4}} T^{a_{2}}\right)_{\alpha_{3} \alpha_{1}}}^{\times\left\{-\frac{s^{2}}{M_{\mathrm{s}}^{2}}\left(-\frac{u}{s}\right)^{3 / 2} \frac{1}{s-M_{\mathrm{s}}^{2}+i M_{\mathrm{s}} \Gamma_{q^{*}, 1 \mathrm{st}}^{J=3 / 2}}\right.} \\
& \left.\quad-\frac{s^{3}}{4 M_{\mathrm{s}}^{4}}\left[\frac{3}{5}\left(-\frac{u}{s}\right)^{3 / 2} \frac{1}{s-2 M_{\mathrm{s}}^{2}+i \sqrt{2} M_{\mathrm{s}} \Gamma_{q^{*}, 2 \mathrm{nd}}^{J=3 / 2}}+\frac{2}{5}\left(-\frac{u}{s}\right)^{3 / 2}\left(\frac{5 t+s}{s}\right) \frac{1}{s-2 M_{\mathrm{s}}^{2}+i \sqrt{2} M_{\mathrm{s}} \Gamma_{q^{*}, 2 \mathrm{nd}}^{J=5 / 2}}\right]\right\} .
\end{aligned}
$$

Spin- and color-averaged squared amplitude including the interference effects is calculated from 
eqs.(A.28)-(A.29) as

$$
\begin{aligned}
& \left|\mathcal{M}_{1 \text { st }+2 \text { nd }}(q g \rightarrow q g)\right|^{2} \\
& =\frac{4}{9} g^{4} \times\left\{\frac{s}{M_{\mathrm{s}}^{4}}\left[\frac{s^{2}(-u)}{\left(s-M_{\mathrm{s}}^{2}\right)^{2}+\left(M_{\mathrm{S}} \Gamma_{q^{*}, 1 \mathrm{st}}^{J=1 / 2}\right)^{2}}+\frac{(-u)^{3}}{\left(s-M_{\mathrm{s}}^{2}\right)^{2}+\left(M_{\mathrm{S}} \Gamma_{q^{*}, 1 \mathrm{st}}^{J=3 / 2}\right)^{2}}\right]\right. \\
& +\frac{s^{3}}{16 M_{\mathrm{S}}^{8}}\left[\frac{1}{9} \frac{s^{2}(-u)}{\left(s-2 M_{\mathrm{S}}^{2}\right)^{2}+\left(\sqrt{2} M_{\mathrm{S}} \Gamma_{q^{*}, 2 \text { nd }}^{J=1 / 2}\right)^{2}}+\frac{9}{25} \frac{(-u)^{3}}{\left(s-2 M_{\mathrm{S}}^{2}\right)^{2}+\left(\sqrt{2} M_{\mathrm{s}} \Gamma_{q^{*}, 2 \text { nd }}^{J=3 / 2}\right)^{2}}\right] \\
& +\frac{s}{4 M_{\mathrm{S}}^{8}}\left[\frac{1}{9} \frac{s^{2}(-u)(3 t+s)^{2}}{\left(s-2 M_{\mathrm{s}}^{2}\right)^{2}+\left(\sqrt{2} M_{\mathrm{s}} \Gamma_{q^{*}, 2 \mathrm{nd}}^{J=3 / 2}\right)^{2}}+\frac{1}{25} \frac{(-u)^{3}(5 t+s)^{2}}{\left(s-2 M_{\mathrm{s}}^{2}\right)^{2}+\left(\sqrt{2} M_{\mathrm{S}} \Gamma_{q^{*}, 2 \mathrm{nd}}^{J=5 / 2}\right)^{2}}\right] \\
& +\frac{s^{2}}{2 M_{\mathrm{s}}^{6}}\left[\frac{1}{3} \frac{s^{2}(-u)}{\left(s-M_{\mathrm{s}}^{2}\right)^{2}+\left(M_{\mathrm{s}} \Gamma_{q^{*}, 1 \mathrm{st}}^{J=1 / 2}\right)^{2}} \frac{\left(s-M_{\mathrm{s}}^{2}\right)\left(s-2 M_{\mathrm{s}}^{2}\right)+\sqrt{2} M_{\mathrm{s}}^{2} \Gamma_{q^{*}, 1 \mathrm{st}}^{J=1 / 2} \Gamma_{q^{*}, 2 \mathrm{nd}}^{J=1 / 2}}{\left(s-2 M_{\mathrm{s}}^{2}\right)^{2}+\left(\sqrt{2} M_{\mathrm{s}} \Gamma_{q^{*}, 2 \text { nd }}^{J=1 / 2}\right)^{2}}\right. \\
& \left.+\frac{3}{5} \frac{(-u)^{3}}{\left(s-M_{\mathrm{s}}^{2}\right)^{2}+\left(M_{\mathrm{s}} \Gamma_{q^{*}, 1 \mathrm{st}}^{J=3 / 2}\right)^{2}} \frac{\left(s-M_{\mathrm{s}}^{2}\right)\left(s-2 M_{\mathrm{s}}^{2}\right)+\sqrt{2} M_{\mathrm{s}}^{2} \Gamma_{q^{*}, 1 \mathrm{st}}^{J=3 / 2} \Gamma_{q^{*}, 2 \text { nd }}^{J=3 / 2}}{\left(s-2 M_{\mathrm{s}}^{2}\right)^{2}+\left(\sqrt{2} M_{\mathrm{s}} \Gamma_{q^{*}, 2 \text { nd }}^{J=3 / 2}\right)^{2}}\right] \\
& +\frac{s}{M_{\mathrm{s}}^{6}}\left[\frac{1}{3} \frac{s^{2}(-u)(3 t+s)}{\left(s-M_{\mathrm{s}}^{2}\right)^{2}+\left(M_{\mathrm{s}} \Gamma_{q^{*}, 1 \mathrm{st}}^{J=1 / 2}\right)^{2}} \frac{\left(s-M_{\mathrm{s}}^{2}\right)\left(s-2 M_{\mathrm{s}}^{2}\right)+\sqrt{2} M_{\mathrm{s}}^{2} \Gamma_{q^{*}, 1 \mathrm{st}}^{J=1 / 2} \Gamma_{q^{*}, 2 \mathrm{nd}}^{J=3 / 2}}{\left(s-2 M_{\mathrm{s}}^{2}\right)^{2}+\left(\sqrt{2} M_{\mathrm{s}} \Gamma_{q^{*}, 2 \mathrm{nd}}^{J=3 / 2}\right)^{2}}\right. \\
& \left.+\frac{1}{5} \frac{(-u)^{3}(5 t+s)}{\left(s-M_{\mathrm{s}}^{2}\right)^{2}+\left(M_{s} \Gamma_{q^{*}, 1 \mathrm{st}}^{J=3 / 2}\right)^{2}} \frac{\left(s-M_{\mathrm{s}}^{2}\right)\left(s-2 M_{\mathrm{s}}^{2}\right)+\sqrt{2} M_{\mathrm{s}}^{2} \Gamma_{q^{*}, 1 \mathrm{st}}^{J=3 / 2} \Gamma_{q^{*}, 2 \mathrm{nd}}^{J=5 / 2}}{\left(s-2 M_{\mathrm{s}}^{2}\right)^{2}+\left(\sqrt{2} M_{\mathrm{s}} \Gamma_{q^{*}, 2 \text { nd }}^{J=5 / 2}\right)^{2}}\right] \\
& +\frac{s^{2}}{4 M_{\mathrm{s}}^{8}}\left[\frac{1}{9} \frac{s^{2}(-u)(3 t+s)}{\left(s-2 M_{\mathrm{s}}^{2}\right)^{2}+\left(\sqrt{2} M_{\mathrm{s}} \Gamma_{q^{*}, 2 \text { nd }}^{J=1 / 2}\right)^{2}} \frac{\left(s-2 M_{\mathrm{s}}^{2}\right)^{2}+2 M_{s}^{2} \Gamma_{q^{*}, 2 \text { nd }}^{J=1 / 2} \Gamma_{q^{*}, 2 \text { nd }}^{J=3 / 2}}{\left(s-2 M_{\mathrm{s}}^{2}\right)^{2}+\left(\sqrt{2} M_{\mathrm{s}} \Gamma_{q^{*}, 2 \text { nd }}^{J=3 / 2}\right)^{2}}\right. \\
& \left.\left.+\frac{3}{25} \frac{(-u)^{3}(5 t+s)}{\left(s-2 M_{\mathrm{s}}^{2}\right)^{2}+\left(\sqrt{2} M_{\mathrm{s}} \Gamma_{q^{*}, 2 \mathrm{nd}}^{J=3 / 2}\right)^{2}} \frac{\left(s-2 M_{\mathrm{s}}^{2}\right)^{2}+2 M_{\mathrm{s}}^{2} \Gamma_{q^{*}, 2 \mathrm{nd}}^{J=3 / 2} \Gamma_{q^{*}, 2 \mathrm{nd}}^{J=5 / 2}}{\left(s-2 M_{\mathrm{s}}^{2}\right)^{2}+\left(\sqrt{2} M_{\mathrm{s}} \Gamma_{q^{*}, 2 \mathrm{nd}}^{J=5 / 2}\right)^{2}}\right]\right\} .
\end{aligned}
$$

\section{References}

[1] I. Antoniadis, "A Possible new dimension at a few TeV," Phys. Lett. B 246, 377 (1990).

[2] I. Antoniadis, N. Arkani-Hamed, S. Dimopoulos and G. R. Dvali, "New dimensions at a millimeter to a Fermi and superstrings at a TeV," Phys. Lett. B 436, 257 (1998) arXiv:hep-ph/9804398].

[3] D. Lust, "Seeing through the String Landscape - a String Hunter's Companion in Particle Physics and Cosmology," JHEP 0903, 149 (2009) [arXiv:0904.4601 [hep-th]].

[4] M. Cicoli, C. P. Burgess and F. Quevedo, "Anisotropic Modulus Stabilisation: Strings at LHC Scales with Micron-sized Extra Dimensions," arXiv:1105.2107 [hep-th].

[5] D. Lust, S. Stieberger and T. R. Taylor, "The LHC String Hunter's Companion," Nucl. Phys. B 808, 1 (2009) [arXiv:0807.3333 [hep-th]].

[6] M. Berkooz, M. R. Douglas and R. G. Leigh, "Branes intersecting at angles," Nucl. Phys. B 480, 265 (1996) arXiv:hep-th/9606139]. 
[7] L. A. Anchordoqui, H. Goldberg, D. Lust, S. Nawata, S. Stieberger and T. R. Taylor, "LHC Phenomenology for String Hunters,” Nucl. Phys. B 821, 181 (2009) arXiv:0904.3547 [hep-ph]].

[8] L. A. Anchordoqui, H. Goldberg, D. Lust, S. Nawata, S. Stieberger and T. R. Taylor, "Dijet signals for low mass strings at the LHC," Phys. Rev. Lett. 101, 241803 (2008) arXiv:0808.0497 [hep-ph]].

[9] N. Kitazawa, "A Closer look at string resonances in dijet events at the LHC," JHEP 1010, 051 (2010). arXiv:1008.4989 [hep-ph]].

[10] S. Cullen, M. Perelstein and M. E. Peskin, "TeV strings and collider probes of large extra dimensions," Phys. Rev. D 62, 055012 (2000) arXiv:hep-ph/0001166.

[11] L. A. Anchordoqui, H. Goldberg and T. R. Taylor, "Decay widths of lowest massive Regge excitations of open strings," Phys. Lett. B 668, 373 (2008) [arXiv:0806.3420 [hep-ph]].

[12] A. Pukhov et al., "CompHEP: A Package for evaluation of Feynman diagrams and integration over multiparticle phase space," arXiv:hep-ph/9908288, and the web page of http://theory.sinp.msu.ru/ pukhov/calchep.html.

[13] T. Sjostrand, S. Mrenna and P. Z. Skands, "A Brief Introduction to PYTHIA 8.1," Comput. Phys. Commun. 178, 852 (2008) arXiv:0710.3820 [hep-ph]].

[14] S. Ovyn, X. Rouby and V. Lemaitre, "DELPHES, a framework for fast simulation of a generic collider experiment," arXiv:0903.2225 [hep-ph].

[15] R. Brun and F. Rademakers, "ROOT: An object oriented data analysis framework," Nucl. Instrum. Meth. A 389, 81 (1997).

[16] http://chercher.phys.se.tmu.ac.jp/StringEventsCalcHEP.html

[17] S. Chatrchyan et al. [CMS Collaboration], "Search for Resonances in the Dijet Mass Spectrum from 7 TeV pp Collisions at CMS," Phys. Lett. B 704, 123 (2011) arXiv:1107.4771 [hep-ex]].

[18] G. Aad et al. [ATLAS Collaboration], "Search for New Physics in Dijet Mass and Angular Distributions in pp Collisions at $\sqrt{s}=7$ TeV Measured with the ATLAS Detector," New J. Phys. 13, 053044 (2011) [arXiv:1103.3864 [hep-ex]].

[19] N. Boelaert and T. Akesson, "Dijet angular distributions at $\sqrt{s}=14$ TeV," Eur. Phys. J. C 66, 343 (2010) [arXiv:0905.3961 [hep-ph]].

[20] G. Cowan, "Statistics." on pages 320-329 of "Review of Particle Physics," Phys. Lett. B 667, 1 (2008).

[21] Z. Dong, T. Han, M. x. Huang and G. Shiu, "Top Quarks as a Window to String Resonances," JHEP 1009, 048 (2010) [arXiv:1004.5441 [hep-ph]]. 